\title{
Synthesis and properties of fluorescent 1,3-substituted mono and biindolizines
}

\author{
Alexandru Rotaru, ${ }^{\mathrm{a}}$ Ioan Druta, ${ }^{\mathrm{a}}$ Ecaterina Avram, ${ }^{\mathrm{b}}$ and Ramona Danac ${ }^{\mathrm{a}, *}$ \\ ${ }^{a}$ Faculty of Chemistry, "Al. I. Cuza” University of Iasi, 11 Carol I, Iasi 700506, Romania \\ b “Petru Poni” Institute of Macromolecular Chemistry, 41A Grigore Ghica Voda Alley, \\ Iasi 700487, Romania \\ E-mail: rdanac@uaic.ro
}

\begin{abstract}
Substituted mono and biindolizines were synthesized in good yields via 3+2 dipolar cycloaddition of pyridinium ylides generated in situ from suitable 1-(2-oxoethyl)pyridinium bromides. The route allows the introduction a variety of substituents at positions 1 and 3 ( 1 ' and 3' for biindolizines) of the indolizine ring. All the synthesized compounds display pronounced fluorescence and detailed studies of the fluorescence properties in different solvents, as well as in acidic conditions for compounds 3a-e, were investigated.
\end{abstract}

Keywords: Pyridinium ylides, 3+2 cycloaddition, indolizines, fluorescence

\section{Introduction}

Indolizine is an aromatic bicyclic $10 \pi$-electron system and constitutional isomer of $1 \mathrm{H}$-indole which, due to its special electronic structure, has long drawn much theoretical interest. ${ }^{1,2}$ Additionally, indolizine derivatives have been found to possess a variety of biological activities such as anti-inflammatory, ${ }^{3}$ antiviral, ${ }^{4}$ analgesic ${ }^{5}$ and antitumor ${ }^{6}$ activities. All these make indolizines an important synthetic target in view of developing new pharmaceuticals for the treatment of cancer, ${ }^{6}$ cardiovascular diseases, ${ }^{3 \mathrm{~b}, 7}$ and HIV infections. ${ }^{8}$ In addition, polycyclic indolizine and biindolizine derivatives have been found to have long wavelength absorption and strong fluorescence in the visible region. ${ }^{9}$ Considering these important properties and the steadily increasing importance of fluorophores in biolabeling and environmental trace analysis, the synthesis of these types of compounds has drawn the interest of chemists to develop new drugs, novel classes of fluorescent dyes or biological markers.

However, investigation of the biological activities, fluorescence and electric properties of indolizines is still impeded by the lack of efficient and general synthetic methods to synthesize indolizines with various functional groups at specific positions. Two general routes for indolizine 
syntheses are known. ${ }^{2 a}$ The first is based on the cyclizing condensation of suitable pyridinium precursors. The second approach takes advantage of a [3+2] cycloaddition of pyridinium ylides with various double or triple bond Michael acceptors. ${ }^{10,11}$

Earlier, our group reported the synthesis of symmetrical and highly fluorescent biindolizines by the reaction of in situ generated symmetrical bis-ylides with activated alkynes. ${ }^{12 a}$ Unsymmetrical indolizines were also synthesized by our group using unsymmetrical bisylides. ${ }^{12 \mathrm{c}}$ Use of these methods in labeling was limited, due to the symmetrical design and the impossibility of having only one labeled group. Here we report the synthesis and fluorescent properties of new substituted monoindolizines by [3+2] cycloaddition of pyridinium ylides with various activated alkynes as well as a new synthesis pathway to new unsymmetrically substituted biindolizines from monoindolizines, in order to increase the degree of asymmetry and also to investigate the possibility of introducing various substituents into the biindolizine ring.

\section{Results and Discussion}

\section{Synthesis}

The synthesis and characterization of pyridinium monoquaternary salts of 4,4'-bipyridine were reported earlier by our group. ${ }^{12 c, d}$ Such quaternary salts were used as starting materials for the indolizine syntheses described in this paper. First, the reactions of the $N$-ylides derived from the quaternary pyridinium salts $\mathbf{1}$, with ethyl propiolate 2 were investigated (Scheme 1). Thus, 1-(2oxoethyl)pyridinium bromide $1(1.0 \mathrm{mmol})$ was deprotonated with triethylamine $(3.0 \mathrm{mmol})$ to give the resonance stabilized pyridinium ylide in situ, an allyl-type 1,3-dipole, that readily underwent 1,3-dipolar cycloaddition with $2(1.0 \mathrm{mmol})$ to give an intermediate dihydroindolizine as the expected cycloadduct. In agreement with the literature, ${ }^{9 f, 12}$ only indolizines $\mathbf{3}$ were isolated as a consequence of oxidative aromatization. These products are fully characterized by spectral (IR, ${ }^{1} \mathrm{H}$ and ${ }^{13} \mathrm{C} \mathrm{NMR}$, and MS) and analytical data. Notably, the ${ }^{1} \mathrm{H}$ NMR spectra of 1a-e were characterized by protons of the ester group (a triplet at $\delta=1.34-1.44 \mathrm{ppm}$ and a quartet at $\delta=$ $3.85-4.42)$ and an unusually low field absorption $(\delta=9.95-10.03 \mathrm{ppm})$ of the proton at C5 of indolizine ring caused by the strong anisotropic deshielding effect of the nearby carbonyl group. 

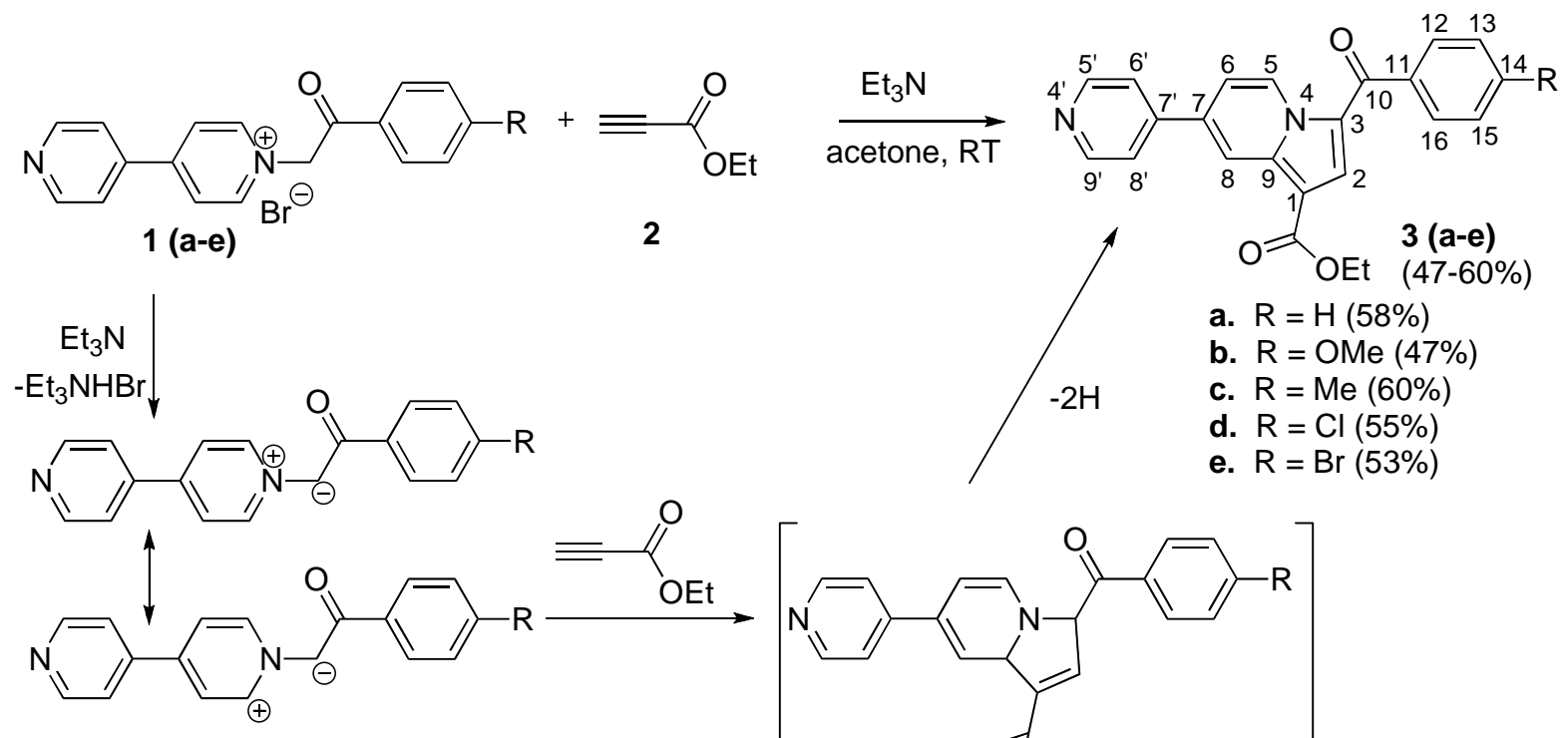
a. $\mathrm{R}=\mathrm{H}(58 \%)$
b. $\mathrm{R}=\mathrm{OMe}(47 \%)$
c. $\mathrm{R}=\mathrm{Me}(60 \%)$
d. $\mathrm{R}=\mathrm{Cl}(55 \%)$
e. $R=\operatorname{Br}(53 \%)$

Scheme 1. Synthesis of 7-pyridylindolizines by $3+2$ dipolar cycloaddition of in situ generated ylides with ethyl propiolate.

Secondly, the synthesis of highly substituted, unsymmetrical indolizines was studied (Scheme 2). The pyridyl-indolizine 3 (1 $\mathrm{mmol})$ was subjected to the reaction with bromoacetophenones $4(1.1 \mathrm{mmol})$ in anhydrous acetone to achieve quaternization of the pyridine giving the indolizinyl-pyridinium salts 5 in very good yields (Table 1). These compounds were characterized by spectral (IR and ${ }^{1} \mathrm{H}$ ) and analytical data. Proton NMR spectroscopy showed a characteristically low field absorption of an indolizine proton at $\mathrm{C} 5^{\prime}(\delta=$ $9.56-10.05 \mathrm{ppm})$ and of the two protons at positions 3 and 5 of the pyridine ring $(\delta=9.37-$ $9.46 \mathrm{ppm})$. The methylene protons adjacent to the quaternary nitrogen, were also very deshielded, showing signals at $\delta=7.14-7.29 \mathrm{ppm}$. In the IR spectra there were three absorption bands corresponding to the three $\mathrm{C}=\mathrm{O}$ groups: one for the conjugated ester at $1698-1707 \mathrm{~cm}^{-1}$ and the other two for the ketone $\mathrm{C}=\mathrm{O}$ bonds at $1641-1687 \mathrm{~cm}^{-1}$ and $1608-1644 \mathrm{~cm}^{-1}$.

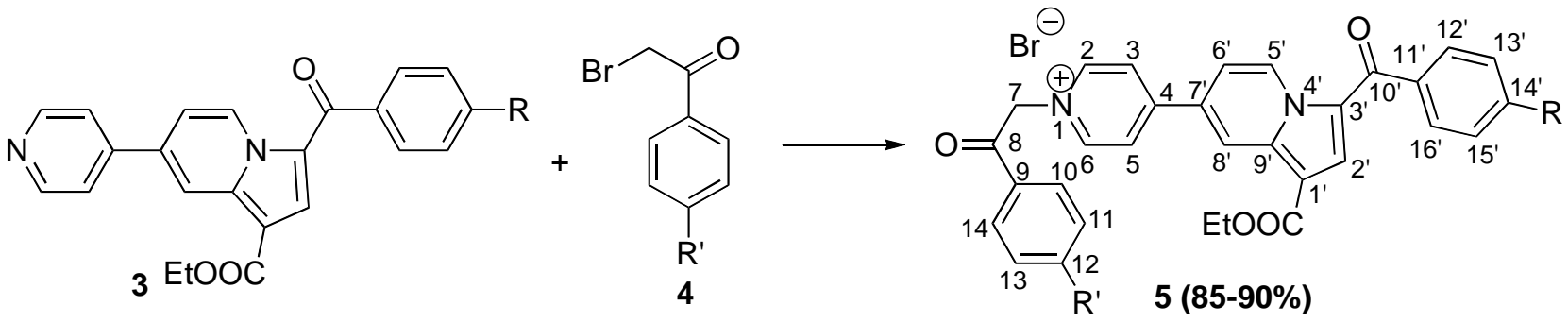

Scheme 2. Synthesis of indolizinyl-pyridinium quaternary salts by quaternization with bromoacetophenone. 
Table 1. Synthesis of indolizinyl-pyridinium quaternary salts 5a-e

\begin{tabular}{|c|c|c|}
\hline Indolizine 3 & Bromoacetophenone 4 & Indolizinyl-pyridinium salt $\mathbf{5}$ (yield) ${ }^{\mathrm{a}}$ \\
\hline $3 \mathbf{a}, \mathrm{R}=\mathrm{H}$ & $4 \mathbf{a}, \mathrm{R}^{\prime}=\mathrm{OMe}$ & $\mathbf{5} \mathbf{a},(88 \%)$ \\
\hline $3 \mathbf{b}, \mathrm{R}=\mathrm{OMe}$ & $4 \mathbf{b}, \mathrm{R}^{\prime}=\mathrm{Br}$ & $5 \mathbf{b},(85 \%)$ \\
\hline $3 \mathbf{c}, \mathrm{R}=\mathrm{Me}$ & $4 \mathbf{c}, \mathrm{R}^{\prime}=\mathrm{NO}_{2}$ & 5 c, $(87 \%)$ \\
\hline $3 \mathbf{d}, \mathrm{R}=\mathrm{Cl}$ & $4 \mathbf{d}, \mathrm{R}^{\prime}=\mathrm{Me}$ & $5 \mathrm{~d},(90 \%)$ \\
\hline $3 \mathbf{e}, \mathrm{R}=\mathrm{Br}$ & $4 \mathbf{e}, \mathrm{R}^{\prime}=\mathrm{Cl}$ & 5 e, $(86 \%)$ \\
\hline
\end{tabular}

${ }^{a}$ Isolated yields after column chromatography.

Using the indolizinyl-pyridinium quaternary salts $\mathbf{5}$, five new unsymmetrical biindolizines were synthesized using methyl propiolate (Scheme 3). Thus, compounds $5(1.0 \mathrm{mmol})$ were suspended in THF together with equivalent amount of methyl propiolate $(1.0 \mathrm{mmol})$ and then triethylamine $(3.0 \mathrm{mmol})$ was added dropwise. As in the mechanism shown in Scheme 1, methyl propiolate reacted with in situ generated pyridinium ylides to give in good yields of the biindolizines 6 through oxidative aromatization of the intermediate dihydroindolizine. The ${ }^{1} \mathrm{H}-$ NMR spectra of the biindolizines showed signals for the new methyl ester group as singlets at $3.95 \mathrm{ppm}$ and integration of the indolizine protons showed the presence of two such units. The IR spectra of these compounds showed absorption bands for the ester and ketone $\mathrm{C}=\mathrm{O}$ groups.

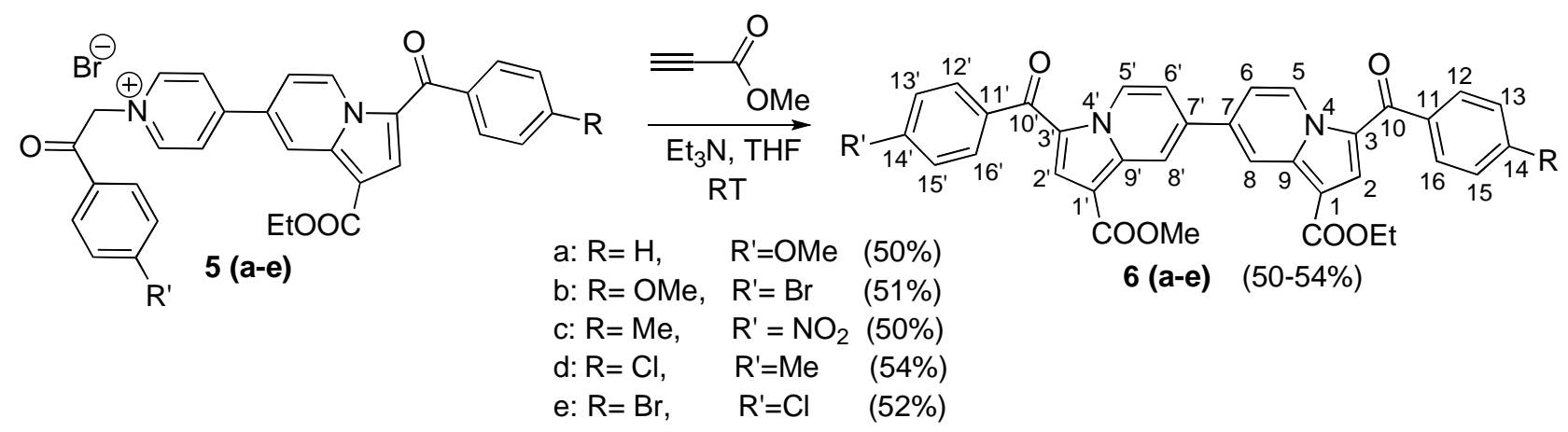

Scheme 3. Synthesis of unsymmetrical biindolizines by $3+2$ dipolar cycloaddition of in situ generated indolizinyl-pyridinium ylides with methyl propiolate.

\section{Fluorescence measurements}

As shown in previous studies ${ }^{9 \mathrm{~d}-\mathrm{f}}$ some indolizines and biindolizines with related structure are highly fluorescent (some of the biindolizines having a remarkably high quantum yield). ${ }^{9 \mathrm{~d}, \mathrm{e}}$ Thus the UV/VIS and fluorescence properties of compound 3a-e in several solvents were investigated. Absorption maxima for all investigated compounds do not show big differences in cyclohexane, toluene, dichloromethane or ethanol, varying between 376 and $384 \mathrm{~nm}$. In fluorescence spectra, maxima for compounds 3a-e vary between 443 and $452 \mathrm{~nm}$ with a characteristic shoulder around $510 \mathrm{~nm}$ (Figure 1, blue curve). 
The presence of a basic pyridine nitrogen atom in the 7-pyridyl indolizines 3a-e suggested that there might be a $\mathrm{pH}$-dependence of the emission properties similar to previously reported compounds. ${ }^{9 f}$ To investigate the influence of the pyridine nitrogen atom, compound 3a was dissolved in dichloromethane and the emission spectrum was recorded. Then, an excess of concentrated hydrochloric acid was added then the emission spectrum was recorded again (Figure 1, red curve). After protonation, a shift of the fluorescence maxima into the yellow region of the spectrum and a significant increase of fluorescence was observed.

To characterize compounds 3a-e on the basis of absorption-emission properties, Stokes shifts for both non-protonated and protonated forms were calculated (Table 2). The data show slightly higher Stokes shifts in the protonated form in comparison to the non-protonated species, for all the investigated compounds.
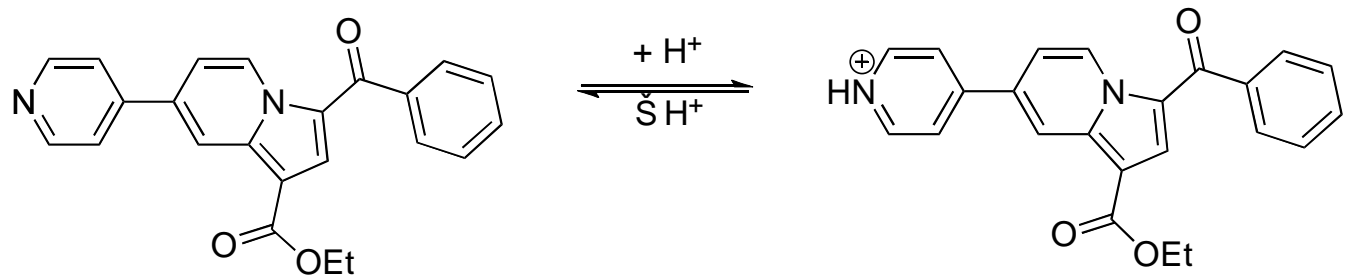

$\lambda_{\max , \mathrm{em}}=448 \mathrm{~nm}$

$$
\lambda_{\max , \mathrm{em}}=495 \mathrm{~nm}
$$

Schematic representation of equilibrium of protonation-deprotonation for the compound 3a.

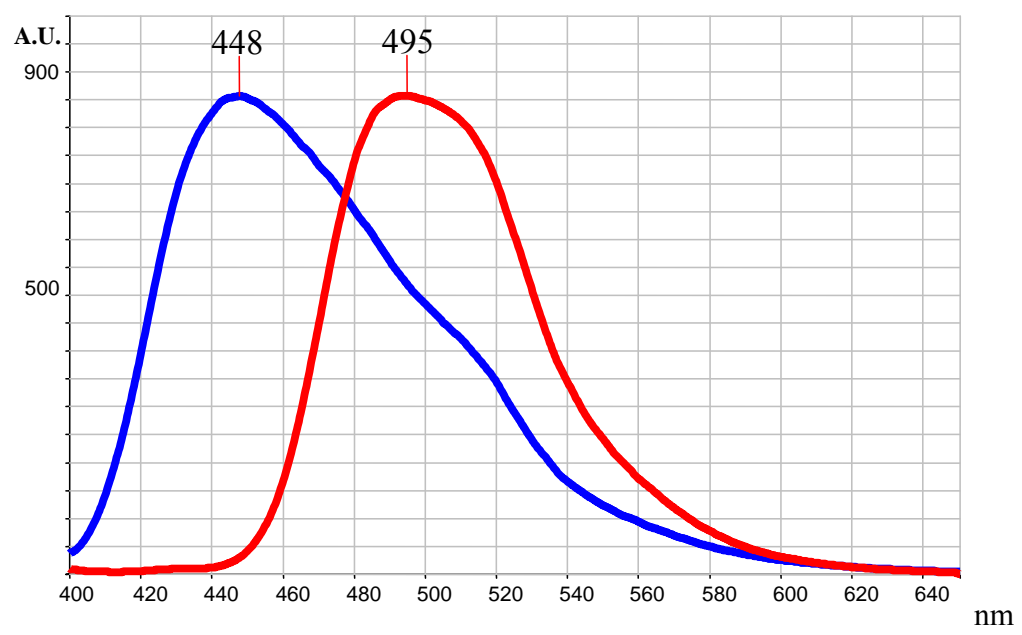

Figure 1. Fluorescence spectra of compound 3a in dichloromethane (blue curve) and mixture of dichloromethane and concentrated hydrochloric acid (red curve) with the emission maxima at $448 \mathrm{~nm}$ for non protonated form and $495 \mathrm{~nm}$ for the protonated form. 
Table 2. Maxima of absorption and emission and Stokes shifts, calculated for non-protonated and protonated forms of compounds 3a-e in dichloromethane

\begin{tabular}{cccccc}
\hline $\begin{array}{c}\text { Com } \\
\text { pound }\end{array}$ & $\begin{array}{c}\text { Absorption } \\
\text { (nonprotonated) } \\
\lambda_{\text {max, abs }}[\mathrm{nm}]\end{array}$ & $\begin{array}{c}\text { Absorption } \\
\text { (protonated) } \\
\lambda_{\max , \text { abs }}[\mathrm{nm}]\end{array}$ & $\begin{array}{c}\text { Emission } \\
\text { (nonprotonated) }\end{array}$ & $\begin{array}{c}\text { Emission } \\
\text { (protonated) } \\
\lambda_{\max , \mathrm{em}}[\mathrm{nm}]\end{array}$ & $\begin{array}{c}\text { Stokes shift, } \\
\text { nonprotonated/ } \\
\text { protonated } \\
\lambda_{\max , \text { em }}[\mathrm{nm}]\end{array}$ \\
\hline 3 a & 382 & - & 448 & 495 & $\left.38 \mathrm{~cm}^{-1}\right]$ \\
3 b & 381 & 419 & 443 & 500 & $3680 / 3870$ \\
3 c & 383 & 413 & 449 & 496 & $3840 / 4050$ \\
3 d & 384 & 412 & 447 & 494 & $3670 / 4030$ \\
3 e & 386 & 410 & 451 & 492 & $3730 / 4070$ \\
\hline
\end{tabular}

${ }^{\mathrm{a}} \Delta \widetilde{v}=1 / \lambda_{\max , \text { abs }}-1 / \lambda_{\max , \text { em }}\left[\mathrm{cm}^{-1}\right]$. Values calculated by this equation are apparent and could give errors of $5-20 \%{ }^{13}$

Absorption and emission spectra for compounds 6a-e were measured in dichloromethane (Figure 2) and the results are presented in Table 2. The fluorescence spectra showed maxima around 465 $\mathrm{nm}$ which is similar to previously reported biindolizines. ${ }^{9 \mathrm{~d}-\mathrm{f}}$ Further investigation of the solvent effects, quantum yields and influences of different substituents in the mono and biindolizine systems are underway.

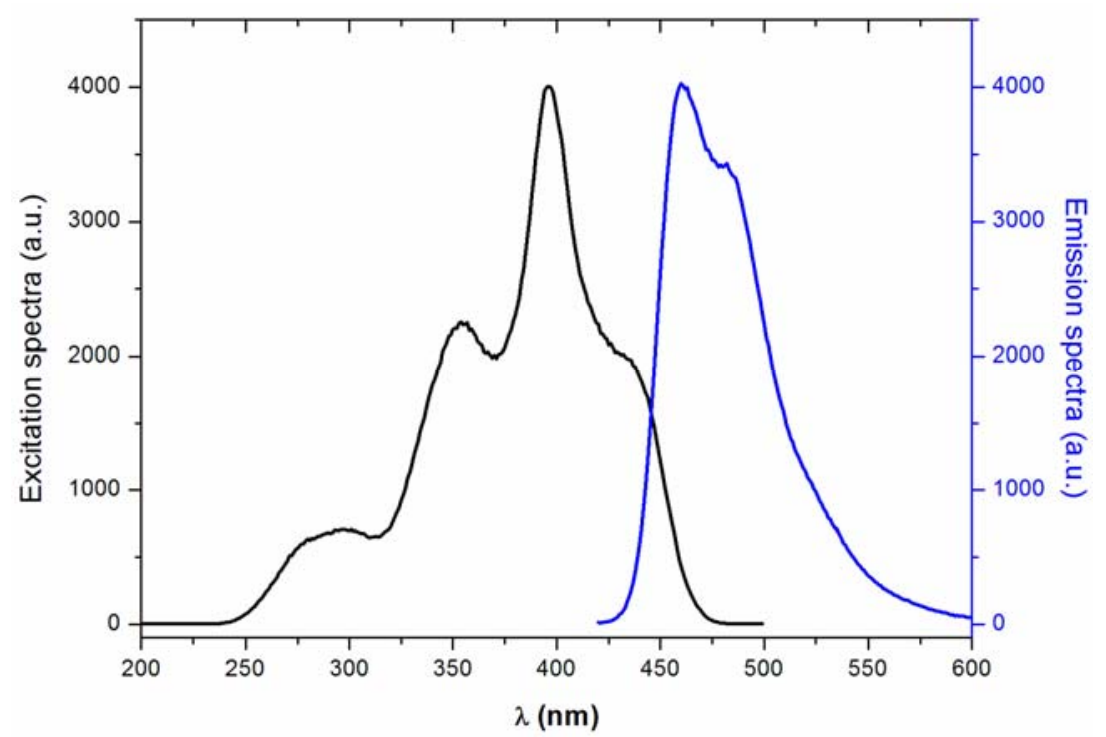

Figure 2. Absorption spectrum (black curve) with maximum at $399 \mathrm{~nm}$ and emission spectra (blue curve) with maximum at $460 \mathrm{~nm}$ of compound $\mathbf{6 a}$ in dichloromethane. 
Table 2. Maxima of absorption and emission and Stokes shifts, calculated for non-protonated and protonated forms of compounds 3a-e in dichloromethane.

\begin{tabular}{cccc}
\hline $\begin{array}{c}\text { Com } \\
\text { pound }\end{array}$ & $\begin{array}{c}\text { Absorption } \\
\lambda_{\max , \text { abs }}[\mathrm{nm}]\end{array}$ & $\begin{array}{c}\text { Emission } \\
\lambda_{\max , \mathrm{em}}[\mathrm{nm}]\end{array}$ & $\begin{array}{c}\text { Stokes shift, }{ }^{\text {a }} \\
\Delta \widetilde{\nu}\left[\mathrm{cm}^{-1}\right]\end{array}$ \\
\hline 6a & 399 & 460 & 3860 \\
6b & 409 & 475 & $3680 / 3870$ \\
6c & 407 & 465 & $3840 / 4050$ \\
6d & 396 & 460 & $3670 / 4030$ \\
6e & 402 & 465 & $3730 / 4070$ \\
\hline
\end{tabular}

${ }^{\mathrm{a}} \Delta \widetilde{v}=1 / \lambda_{\max , \text { abs }}-1 / \lambda_{\max , \mathrm{em}}\left[\mathrm{cm}^{-1}\right]$.

In conclusion, we have successfully synthesized monoindolizines and biindolizines in good yields using 3+2 dipolar cycloadditions of pyridinium ylides generated in situ from suitable 1-(2oxoethyl)pyridinium bromides. The route allows the introduction of substituents at positions 1 and 3 ( 1 ' and 3 ' for biindolizines) of the indolizine ring. All the new synthesized compounds displayed pronounced fluorescence and detailed studies of the fluorescence properties in dichloromethane for compounds 3a-e and 6a-e were made. Further studies will be mostly directed to investigations of the fluorescence and electrical properties of all the synthesized compounds in view of their direct relevance in biomolecule labeling and/or in the semiconductors field.

\section{Experimental Section}

General. Melting points were recorded on a Meltemp II melting point apparatus and are uncorrected. Proton and carbon nuclear magnetic resonance $\left(\delta_{\mathrm{H}}, \delta_{\mathrm{C}}\right)$ spectra were recorded on Bruker Avance 400 DRX or Bruker ARX 300 spectrometers. All chemical shifts are quoted on the $\delta$-scale in ppm using TMS or residual solvents $\left(\mathrm{CHCl}_{3}, \mathrm{DMSO}\right)$ as internal standards. Coupling constants are given in Hz. IR spectra were recorded on a FTIR Shimadzu spectrometer. Low resolution mass spectra were recorded on a Finnigan MAT 90 and MAT 95 Q; 70 eV, FAB in the positive mode; $\mathrm{m} / \mathrm{z}$ (rel. int. in \%). Thin layer chromatography (TLC) was carried out on Merck silica gel $60 \mathrm{~F}_{254}$ plates. Visualisation of the plates was achieved using a UV lamp $\left(\lambda_{\max }=\right.$ 254 or $365 \mathrm{~nm}$ ). UV/vis spectra were measured using Perkin Elmer Models Lambda 16; Fluorescence spectra with Perkin Elmer LS-55 and spectrofluorimeter SLM-8000. Compounds 3a-e and 6a-e were dissolved in dichloromethane to the concentration of $10 \mu \mathrm{M}$ and measured in $1 \mathrm{~cm}$ fluorescence cuvettes.

\section{General procedure for preparation of 3a-e compounds}


The cycloimmonium salt 1a-e ( 1 mmol, 1 equiv., $0.36 \mathrm{~g}$ 1a, $0.39 \mathrm{~g} \mathbf{1 b}, 0.37 \mathrm{~g} \mathbf{1 c}, 0.39 \mathrm{~g} \mathbf{1 d}, 0.43$ g 1e) and ethyl propiolate ( $1.1 \mathrm{mmol}, 1.1$ equiv., $0.11 \mathrm{~g})$ were added to $10 \mathrm{~mL}$ of anhydrous acetone and the obtained suspension stirred at room temperature. A solution of triethylamine (TEA) (3 mmol, 3 equiv., $0.30 \mathrm{~g})$ in anhydrous acetone $(3 \mathrm{~mL})$ was added dropwise over $2 \mathrm{~h}$ (magnetic stirring) and the resulting mixture was then stirred for 5-6 $\mathrm{h}$ at $\mathrm{rt}$. Water $(10 \mathrm{~mL})$ was added and after stirring for another 15 minutes, the solid was collected by filtration to give a powder which was washed with $10 \mathrm{~mL}$ methanol. The product was recrystallized from ethanolchloroform $(1: 1, \mathrm{v} / \mathrm{v})$.

\section{General procedure for synthesis of quaternary salts $5 a-e$}

A solution of monoindolizine 3 ( 1 mmol, 1 equiv., $0.37 \mathrm{~g} 3 \mathbf{a}, 0.40 \mathrm{~g} 3 \mathbf{b}, 0.38 \mathrm{~g} 3 \mathbf{c}, 0.40 \mathrm{~g}$ 3d, $0.45 \mathrm{~g} 3 \mathbf{e})$ and 2-bromo-4'-X-acetophenone ( $2 \mathrm{mmol}, 2$ equiv.) in anhydrous acetone $(20 \mathrm{~mL})$ was magnetically stirred for 3-4 h at reflux. The resulting bright yellow precipitate was removed by filtration and then washed with acetone. All products were purified by flash chromatography (DCM:MeOH 9:1, v/v).

\section{General procedure for synthesis of biindolizines 6a-e}

The quaternary salt 5 ( 1 mmol, 1 equiv., 0.60 g 5a, 0.68 g 5b, 0.63 g 5c, 0.62 g 5d, 0.68 g 5e) and methyl propiolate ( $1.1 \mathrm{mmol}, 1.1$ equiv., $0.09 \mathrm{~g}$ ) were added to $40 \mathrm{~mL}$ of anhydrous tetrahydrofuran $\mathrm{THF}$ ) and the obtained suspension stirred at room temperature. A solution of triethylamine (TEA) ( 3 mmol, 3 equiv., $0.30 \mathrm{~g})$ in anhydrous tetrahydrofuran $(3 \mathrm{~mL})$ was added dropwise over $2 \mathrm{~h}$ (magnetic stirring) and the resulting mixture was then stirred for 5-6 $\mathrm{h}$ at $\mathrm{rt}$. Water $(15 \mathrm{~mL})$ was added and after stirring for another $15 \mathrm{~min}$, the solid was collected by filtration to give a powder which was washed with $10 \mathrm{~mL}$ methanol. The product was recrystallized from ethanol-chloroform $(1: 4, \mathrm{v} / \mathrm{v})$.

Ethyl 3-benzoyl-7-(pyridin-4-yl)indolizine-1-carboxylate 3a. Yellowish crystals (0.21g, 58\% yield), mp 191-192 ${ }^{\circ} \mathrm{C} .{ }^{1} \mathrm{H}-\mathrm{NMR}\left(\mathrm{CDCl}_{3}, 300 \mathrm{MHz}\right): 9.95$ (1H, d, H-5, J=7.2 Hz,), 8.65-8.69 (3H, m, H-5 ', H-9 ' , H-8), 7.75-7.78 (3H, m, H-12, H-16, H-2), 7.58 (2H, d, H-6 ', H-8 ', J = 5.7 Hz,), 7.44-7.54 (3H, m, H-13, H-14, H-15), 7.28 (1H, dd, H-6, J = 2.1, 7.5 Hz), 4.32 (2H, q, $\left.\mathrm{CH}_{2}, J=7.2 \mathrm{~Hz}\right), 1.35\left(3 \mathrm{H}, \mathrm{t}, \mathrm{CH}_{3}, J=7.2 \mathrm{~Hz}\right) .{ }^{13} \mathrm{C}-\mathrm{NMR}\left(\mathrm{CDCl}_{3}, 75 \mathrm{MHz}\right): 14.5\left(\mathrm{CH}_{3}\right), 60.4$ $\left(\mathrm{CH}_{2}\right), 108.1\left(\mathrm{C}_{\text {quat }}\right), 113.2(\mathrm{CH}), 118.0(\mathrm{CH}), 122.2(\mathrm{CH}), 123.1\left(\mathrm{C}_{\text {quat }}\right), 128.5(\mathrm{CH}), 128.9(\mathrm{CH})$, $129.1(\mathrm{CH}), 129.7(\mathrm{CH}), 131.9(\mathrm{CH}), 139.2\left(\mathrm{C}_{\text {quat }}\right), 139.4\left(\mathrm{C}_{\text {quat }}\right), 147.4(\mathrm{CH}), 163.7\left(\mathrm{C}_{\text {quat }}\right)$, $185.77\left(\mathrm{C}_{\text {quat }}\right)$. MS (70 eV, FAB+): m/z (\%) $371(\mathrm{M}+\mathrm{H}, 100), 370\left(\mathrm{M}^{+}, 28\right)$. IR (KBr, v(cm $\left.{ }^{-1}\right)$ : 1689, 1609, 1287, 1199, 1134, 1078. Anal. Calcd. for $\mathrm{C}_{23} \mathrm{H}_{18} \mathrm{~N}_{2} \mathrm{O}_{3}$ : C, 74.58; H, 4.90; N, 7.56. Found: C, 74.66; H, 4.97; N, 7.75.

Ethyl 3-(4-methoxybenzoyl)-7-(pyridin-4-yl)indolizine-1-carboxylate 3b. Yellowish crystals (0.19g, 47\% yield), mp 186-187 ${ }^{\circ} \mathrm{C} .{ }^{1} \mathrm{H}-\mathrm{NMR}\left(\mathrm{CDCl}_{3}, 300 \mathrm{MHz}\right): 9.89$ (1H, d H-5, J = $\left.7.5 \mathrm{~Hz}\right)$, 8.67-8.69 (3H, m, H-5 ', H-9 ', H-8), 7.78-7.81 (3H, m, H-12, H-16, H-2), 7.62 (2H, d, H-6 ', H$\left.8^{\prime}, J=5.7 \mathrm{~Hz}\right), 7.27(1 \mathrm{H}, \mathrm{dd}, \mathrm{H}-6, J=2.1,7.5 \mathrm{~Hz}), 6.97(2 \mathrm{H}, \mathrm{d}, \mathrm{H}-13, \mathrm{H}-15, J=8.7 \mathrm{~Hz}), 4.34$ $\left(2 \mathrm{H}, \mathrm{q}, \mathrm{CH}_{2}, J=6.9 \mathrm{~Hz}\right), 3.85\left(3 \mathrm{H}, \mathrm{s}, \mathrm{OCH}_{3}\right), 1.36\left(3 \mathrm{H}, \mathrm{t}, \mathrm{CH}_{3}, J=6.9 \mathrm{~Hz}\right) .{ }^{13} \mathrm{C}-\mathrm{NMR}\left(\mathrm{CDCl}_{3}\right.$, 
$75 \mathrm{MHz}): \delta=14.6\left(\mathrm{CH}_{3}\right), 55.5\left(\mathrm{CH}_{3}\right), 60.3\left(\mathrm{CH}_{2}\right), 107.1\left(\mathrm{C}_{\text {quat }}\right), 113.3(\mathrm{CH}), 113,8(\mathrm{CH}), 117.2$ $(\mathrm{CH}), 121.2(\mathrm{CH}), 122.9\left(\mathrm{C}_{\text {quat }}\right), 128.5(\mathrm{CH}), 129.4(\mathrm{CH}), 131.3(\mathrm{CH}), 132.1\left(\mathrm{C}_{\text {quat }}\right), 136.4\left(\mathrm{C}_{\text {quat }}\right)$, $139.4\left(\mathrm{C}_{\text {quat }}\right), 145.3\left(\mathrm{C}_{\text {quat }}\right), 150.6(\mathrm{CH}), 162.7\left(\mathrm{C}_{\text {quat }}\right), 164.0\left(\mathrm{C}_{\text {quat }}\right), 184.6\left(\mathrm{C}_{\text {quat }}\right) . \mathrm{MS}(70 \mathrm{eV}$, FAB +$): m / z(\%) 401(\mathrm{M}+\mathrm{H}, 100), 400\left(\mathrm{M}^{+}, 32\right), 355\left(\mathrm{M}^{+}-\mathrm{C}_{2} \mathrm{H}_{5} \mathrm{O}\right), 328\left(\mathrm{M}^{+}-\mathrm{C}_{3} \mathrm{H}_{5} \mathrm{O}_{2}\right), 293\left(\mathrm{M}^{+}-\right.$ $\left.\mathrm{C}_{7} \mathrm{H}_{7} \mathrm{O}\right)$. IR $\left(\mathrm{KBr}, v\left(\mathrm{~cm}^{-1}\right): 1697,1608,1285,1203,1175,1079\right.$. Anal. Calcd. for $\mathrm{C}_{24} \mathrm{H}_{20} \mathrm{~N}_{2} \mathrm{O}_{4}$ : C, 71.99; H, 5.03; N, 7.00. Found: C, 72.12; H, 5.21; N, 7.15.

Ethyl 3-(4-methylbenzoyl)-7-(pyridin-4-yl)indolizine-1-carboxylate 3c. Yellowish crystals (0.23g, 60\% yield), mp 188-189 ${ }^{\circ} \mathrm{C} .{ }^{1} \mathrm{H}-\mathrm{NMR}\left(\mathrm{CDCl}_{3}, 300 \mathrm{MHz}\right): 10.02(1 \mathrm{H}, \mathrm{dd}, \mathrm{H}-5, J=0.6$, $7.5 \mathrm{~Hz}), 8.75-8.78$ (3H, m, H-5 ', H-9 ', H-8), 7.87 (1H, s, H-2), 7.78 (2H, d, H-12, H-16, J = 8.1 Hz), 7.68 (2H, d, H-6 ' , H-8 ' , J =6.0 Hz), 7.35-7.38 (3H, m, H-13, H-15, H-6), 4.42 (2H, q, $\mathrm{CH}_{2}$ $, J=7.2 \mathrm{~Hz}), 2.49\left(3 \mathrm{H}, \mathrm{s}, \mathrm{CH}_{3}\right), 1.44\left(3 \mathrm{H}, \mathrm{t}, \mathrm{CH}_{3}, J=7.2 \mathrm{~Hz}\right) \cdot{ }^{13} \mathrm{C}-\mathrm{NMR}\left(\mathrm{CDCl}_{3}, 75 \mathrm{MHz}\right): \delta=$ $14.5\left(\mathrm{CH}_{3}\right), 21.6\left(\mathrm{CH}_{3}\right), 60.2\left(\mathrm{CH}_{2}\right), 107,2\left(\mathrm{C}_{\text {quat }}\right), 113.4(\mathrm{CH}), 117.1(\mathrm{CH}), 121.2(\mathrm{CH}), 122.9$ $\left(\mathrm{C}_{\text {quat }}\right), 128.9(\mathrm{CH}), 129.1(\mathrm{CH}), 129.5(\mathrm{CH}), 136.6\left(\mathrm{C}_{\text {quat }}\right), 136.8\left(\mathrm{C}_{\text {quat }}\right), 139.5\left(\mathrm{C}_{\text {quat }}\right), 142.4$ $\left(\mathrm{C}_{\text {quat }}\right), 145.3\left(\mathrm{C}_{\text {quat }}\right), 150.6(\mathrm{CH}), 163.9\left(\mathrm{C}_{\text {quat }}\right), 185.5\left(\mathrm{C}_{\text {quat }}\right) . \mathrm{MS}(70 \mathrm{eV}, \mathrm{FAB}+): \mathrm{m} / \mathrm{z}(\%) 385$ $(\mathrm{M}+\mathrm{H}, 100), 384\left(\mathrm{M}^{+}, 38\right), 355\left(\mathrm{M}^{+}-\mathrm{C}_{2} \mathrm{H}_{5} \mathrm{O}\right), 328\left(\mathrm{M}^{+}-\mathrm{C}_{3} \mathrm{H}_{5} \mathrm{O}_{2}\right), 293\left(\mathrm{M}^{+}-\mathrm{C}_{7} \mathrm{H}_{7} \mathrm{O}\right) . \mathrm{IR}(\mathrm{KBr}$, $v\left(\mathrm{~cm}^{-1}\right): 1699 \mathrm{~cm}^{-1}, 1610,1288,1204,1081,1047$. Anal. Calcd. for $\mathrm{C}_{24} \mathrm{H}_{20} \mathrm{~N}_{2} \mathrm{O}_{3}$ : C, 74.98; $\mathrm{H}$, 5.24; N, 7.29. Found: C, 75.13; H, 5.32; N, 7.54.

Ethyl 3-(4-chlorobenzoyl)-7-(pyridin-4-yl)indolizine-1-carboxylate (3d). Yellowish crystals (0.22g, 55\% yield), mp 215-216 ${ }^{\circ} \mathrm{C} .{ }^{1} \mathrm{H}-\mathrm{NMR}\left(\mathrm{CDCl}_{3}, 300 \mathrm{MHz}\right): 9.93(1 \mathrm{H}, \mathrm{dd}, \mathrm{H}-5, J=0.9$, $7.5 \mathrm{~Hz}$ ), 8.68-8.71 (3H, m, H-5 ', H-9' , H-8), 7.71-7.75 (3H, m, H-12, H-16, H-2), 7.61 (2H, dd, $J=1.5,7.5 \mathrm{~Hz}, \mathrm{H}-6^{\prime}$, H-8 '), 7.45 (2H, d, H-13, H-15, $\left.J=8.7 \mathrm{~Hz}\right), 7.31$ (1H, dd, H-6, $J=1.8$, $7.2 \mathrm{~Hz}), 4.34\left(2 \mathrm{H}, \mathrm{q}, \mathrm{CH}_{2}, J=6.9 \mathrm{~Hz}\right), 1.36\left(3 \mathrm{H}, \mathrm{t}, \mathrm{CH}_{3}, J=6.9 \mathrm{~Hz}\right) .{ }^{13} \mathrm{C}-\mathrm{NMR}\left(\mathrm{CDCl}_{3}, 75\right.$ $\mathrm{MHz}): \delta=14.5\left(\mathrm{CH}_{3}\right), 60.4\left(\mathrm{CH}_{2}\right), 107,8\left(\mathrm{C}_{\text {quat }}\right), 113.6(\mathrm{CH}), 117.5(\mathrm{CH}), 121.8(\mathrm{CH}), 122.5$ $\left(\mathrm{C}_{\text {quat }}\right), 126.7\left(\mathrm{C}_{\text {quat }}\right), 128.9(\mathrm{CH}), 129.6(\mathrm{CH}), 130.6(\mathrm{CH}), 131.7(\mathrm{CH}), 136.2\left(\mathrm{C}_{\text {quat }}\right), 138.1$ $\left(\mathrm{C}_{\text {quat }}\right), 139.7\left(\mathrm{C}_{\text {quat }}\right), 146.6\left(\mathrm{C}_{\text {quat }}\right), 149.1(\mathrm{CH}), 163.8\left(\mathrm{C}_{\text {quat }}\right), 184.4\left(\mathrm{C}_{\text {quat }}\right) . \mathrm{IR}\left(\mathrm{KBr}, \mathrm{v}\left(\mathrm{cm}^{-1}\right)\right.$ : $1699 \mathrm{~cm}^{-1}, 1609,1286,1200,1078$, 1092. Anal. Calcd. for $\mathrm{C}_{23} \mathrm{H}_{17} \mathrm{ClN}_{2} \mathrm{O}_{3}: \mathrm{C}, 68.23 ; \mathrm{H}, 4.23 ; \mathrm{N}$, 6.92; Found: C, 68.35; H, 4.38; N, 7.11.

Ethyl 3-(4-bromobenzoyl)-7-(pyridin-4-yl)indolizine-1-carboxylate (3e). Yellowish crystals (0.24g, 53\% yield), mp 234-235 ${ }^{\circ} \mathrm{C} .{ }^{1} \mathrm{H}-\mathrm{NMR}\left(\mathrm{CDCl}_{3}, 300 \mathrm{MHz}\right): 10.01(1 \mathrm{H}, \mathrm{d}, \mathrm{H}-5, J=7.2$ Hz), 8.77-8.79 (3H, m, H-5 ', H-9 ', H-8), 7.82 (1H, s, H-2), 7.66-7.75 (6H, m, H-13, H-15, H6 ' , H-8 ', H-12, H-16), 7.39 (1H, dd, H-6, J = 2.1, $7.5 \mathrm{~Hz}), 4.42$ (2H, q, $\left.\mathrm{CH}_{2}, J=7.2 \mathrm{~Hz},\right), 1.44$ $\left(3 \mathrm{H}, \mathrm{t}, \mathrm{CH}_{3}, J=7.2 \mathrm{~Hz}\right) .{ }^{13} \mathrm{C}-\mathrm{NMR}\left(\mathrm{CDCl}_{3}, 75 \mathrm{MHz}\right): \delta=14.5\left(\mathrm{CH}_{3}\right), 60.4\left(\mathrm{CH}_{2}\right), 107,9\left(\mathrm{C}_{\text {quat }}\right)$, $113.5(\mathrm{CH}), 117.5(\mathrm{CH}), 121.7(\mathrm{CH}), 122.5\left(\mathrm{C}_{\text {quat }}\right), 126.6\left(\mathrm{C}_{\text {quat }}\right), 128.9(\mathrm{CH}), 129.5(\mathrm{CH}), 130.5$ $(\mathrm{CH}), 131.7(\mathrm{CH}), 136.1\left(\mathrm{C}_{\text {quat }}\right), 138.1\left(\mathrm{C}_{\text {quat }}\right), 139.6\left(\mathrm{C}_{\text {quat }}\right), 146.6\left(\mathrm{C}_{\text {quat }}\right), 149.1(\mathrm{CH}), 163.7$

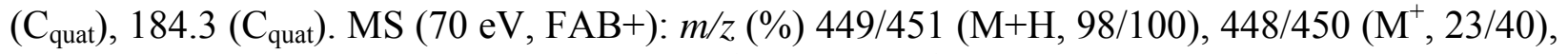
403/405 ( $\left.\mathrm{M}^{+}-\mathrm{C}_{2} \mathrm{H}_{5} \mathrm{O}, 12 / 11\right)$. IR (KBr, $v\left(\mathrm{~cm}^{-1}\right): 1700 \mathrm{~cm}^{-1}, 1610,1288,1201,1076,1076$. Anal. Calcd. for $\mathrm{C}_{23} \mathrm{H}_{17} \mathrm{BrN}_{2} \mathrm{O}_{3}$ : C, 61.48; H, 3.81; N, 6.23; Found: C, 61.60; H, 3.96; N, 6.53.

1-[2-(4-Methoxyphenyl)-2-oxoethyl]-4-[[1-(ethoxycarbonyl)-3-benzoyl]-indolizin-7-

yl]pyridinium bromide 5a. Bright yellow crystals $(0.52 \mathrm{~g}, 88 \%$ yield $), \mathrm{mp} 274-276{ }^{\circ} \mathrm{C} .{ }^{1} \mathrm{H}-$ NMR: 9.56 (1H, d, H-5 ', J = 7.6Hz), 9.37 (2H, d, H-2, H-6, J=6.4 Hz), 8.81 (1H, d, H-8 ' , J =2 
Hz), 8.33 (2H, d, H-3, H-5, J=6.4 Hz), $8.16(2 \mathrm{H}, \mathrm{d}, \mathrm{H}-10, \mathrm{H}-14, J=8.8 \mathrm{~Hz}), 7.81$ (3H, ad, H$12^{\prime}$, H-16 ', H-2 ' ), 7.63 (1H, t, H-14 ', J=7.6 Hz), 7.53 (2H, t, H-13 ', H-15 ' J = 7.6 Hz), 7.46 $(1 \mathrm{H}, \mathrm{dd}, \mathrm{H}-6$ ' $, J=7.6 \mathrm{~Hz}, J=2 \mathrm{~Hz}), 7.15$ (2H, s, H-7), 6.95 (2H, d, H-11, H-13, J =8.8 Hz), 4.42 $\left(2 \mathrm{H}, \mathrm{q}, \mathrm{CH}_{2}, J=7.2 \mathrm{~Hz}\right), 3.84\left(3 \mathrm{H}, \mathrm{s}, \mathrm{OCH}_{3}\right), 1.42\left(3 \mathrm{H}, \mathrm{t}, \mathrm{CH}_{3}, J=7.2 \mathrm{~Hz}\right) . \mathrm{IR}\left(\mathrm{KBr}, \mathrm{v}\left(\mathrm{cm}^{-1}\right)\right.$ : 1706, 1689, 1643, 1241, 1205, 1178. Anal. Calcd. for $\mathrm{C}_{32} \mathrm{H}_{27} \mathrm{BrN}_{2} \mathrm{O}_{5}: \mathrm{C}, 64.11 ; \mathrm{H}, 4.54 ; \mathrm{N}, 4.67$; Found: C, 64.30; H, 4.69; N, 4.79.

\section{1-[2-(4-Bromophenyl)-2-oxoethyl]-4-[[1-(ethoxycarbonyl)-3-(4-methoxybenzoyl)]indolizin-}

7-yl]pyridinium bromide 5b. Yellow crystals $\left(0.57 \mathrm{~g}, 85 \%\right.$ yield), m.p. $260-262{ }^{\circ} \mathrm{C} .{ }^{1} \mathrm{H}-\mathrm{RMN}$ : $9.77(1 \mathrm{H}, \mathrm{d}, \mathrm{H}-5$ ' , J = 7.6 Hz), $9.46(2 \mathrm{H}, \mathrm{d}, \mathrm{H}-3, \mathrm{H}-5, J=6.8 \mathrm{~Hz}), 8.2(1 \mathrm{H}, \mathrm{as}, \mathrm{H}-8$ ' ), 8.35 (2H, d, H-2, H-6, J = 6.8 Hz), 8.00 (2H, d, H-10, H-14, J=8.8 Hz), 7.78 (2H, d, H-12 ', H-16 ' , $J=$ $8.8 \mathrm{~Hz}), 7.72\left(1 \mathrm{H}, \mathrm{s}, \mathrm{H}-2^{\prime}\right), 7.51(2 \mathrm{H}, \mathrm{d}, \mathrm{H}-11, \mathrm{H}-13, J=8.8 \mathrm{~Hz}), 7.47$ (1H, dd, H-6 ', $J=7.6$ $\mathrm{Hz}, J=2 \mathrm{~Hz}), 7.28$ (2H, s, H-7), 6.98 (2H, d, H-13 ', H-15 ', J = 8.8 Hz), 4.40 (2H, q, CH $2, J=$ $7.2 \mathrm{~Hz}, 3.91\left(3 \mathrm{H}, \mathrm{s}, \mathrm{OCH}_{3}\right), 1.40\left(3 \mathrm{H}, \mathrm{t}, \mathrm{CH}_{3}, J=7.2 \mathrm{~Hz}\right)$. IR $\left(\mathrm{KBr}, \mathrm{v}\left(\mathrm{cm}^{-1}\right): 1699,1641,1608\right.$, 1257, 1201, 1170. Anal. Calcd. for $\mathrm{C}_{32} \mathrm{H}_{26} \mathrm{Br}_{2} \mathrm{~N}_{2} \mathrm{O}_{5}$ : C, 56.66; H, 3.86; N, 4.13; Found: C, 56.80; H, 3.99; N, 4.59 .

1-[2-(4-Nitrophenyl)-2-oxoethyl]-4-[[1-(ethoxycarbonyl)-3-(4-methylbenzoyl)]indolizin-7yl]pyridinium bromide 5c. Yellow crystals $(0.54 \mathrm{~g}, 87 \%$ yield $)$, m.p. $260-262{ }^{\circ} \mathrm{C}$. ${ }^{1} \mathrm{H}-\mathrm{NMR}$ : $9.91(1 \mathrm{H}, \mathrm{d}, \mathrm{H}-5$ ',$J=7.2 \mathrm{~Hz}), 9.40(2 \mathrm{H}, \mathrm{d}, \mathrm{H}-3, \mathrm{H}-5, J=6.4 \mathrm{~Hz}), 8.85(1 \mathrm{H}, \mathrm{d}, \mathrm{H}-8$ ',$J=1.8$ $\mathrm{Hz}), 8.35$ (2H, d, H-2, H-6, J = 6.4 Hz), $8.11(2 \mathrm{H}, \mathrm{d}, \mathrm{H}-11, \mathrm{H}-13, J=8.2 \mathrm{~Hz}), 7.87(2 \mathrm{H}, \mathrm{d}, \mathrm{H}-$ $12^{\prime}$, H-16 ', $\left.J=8.7 \mathrm{~Hz}\right), 7.83$ (1H, s, H-2 '), 7.65 (2H, d, H-10, H-14, J=8.2 Hz), 7.44 (1H, dd, H-6 ' $J=7.2 \mathrm{~Hz}, J=1.8 \mathrm{~Hz}), 7.29$ (2H, s, H-7), 7.24 (2H, d, H-13 ', H-15 ', J=8.7 Hz), 4.46 $\left(2 \mathrm{H}, \mathrm{q}, \mathrm{CH}_{2}, J=7.2 \mathrm{~Hz}\right), 2.48\left(3 \mathrm{H}, \mathrm{s}, \mathrm{CH}_{3}\right), 1.49\left(3 \mathrm{H}, \mathrm{t}, \mathrm{CH}_{3}, J=7.2 \mathrm{~Hz}\right) . \mathrm{IR}\left(\mathrm{KBr}, \mathrm{v}\left(\mathrm{cm}^{-1}\right)\right.$ : 1706, 1689, 1644, 1527, 1349, 1235, 1208, 1202. Anal. Calcd. for $\mathrm{C}_{32} \mathrm{H}_{26} \mathrm{BrN}_{3} \mathrm{O}_{6}$ : C, 61.16; $\mathrm{H}$, 4.17; N, 6.69; Found: C, 61.29; H, 4.09; N, 6.90.

1-[2-(4-Methylphenyl)-2-oxoethyl]-4-[[1-(ethoxycarbonyl)-3-(4-chlorobenzoyl)]indolizin-7yl]pyridinium bromide 5d. Yellow crystals (0.55g, 90\% yield), m.p. 268-280 ${ }^{\circ} \mathrm{C}$. ${ }^{1} \mathrm{H}-\mathrm{NMR}$ : 10.05 (1H, d, H-5 ', $J=7.5 \mathrm{~Hz}), 9.46(2 \mathrm{H}, \mathrm{d}, \mathrm{H}-3, \mathrm{H}-5, J=6.9 \mathrm{~Hz}), 8.96(1 \mathrm{H}, \mathrm{d}, \mathrm{H}-8$ ' , $J=1.5$ Hz), 8.35 (2H, d, H-2, H-6, J=6.9 Hz), 8.13 (2H, d, H-12 ' , H-16 ', J=8.4 Hz), 7.87 (1H, s, H2'), 7.83 (2H, d, H-10, H-14, J=8.4 Hz), 7.58 (2H, d, H-13 ', H-15 ', J=8.4Hz), 7,47 (1H, dd, H-6 ' $J=7.5 \mathrm{~Hz}, J=1.5 \mathrm{~Hz}), 7.39$ (2H, d, H-11, H-13, J=8.4 Hz), 7.14 (2H, s, H-7), 4,48 (2H, q, $\left.\mathrm{CH}_{2}, J=7.2 \mathrm{~Hz}\right), 2.48\left(3 \mathrm{H}, \mathrm{s}, \mathrm{CH}_{3}\right), 1.42\left(3 \mathrm{H}, \mathrm{t}, \mathrm{CH}_{3}, J=7.2 \mathrm{~Hz}\right) . \mathrm{IR}\left(\mathrm{KBr}, \mathrm{v}\left(\mathrm{cm}^{-1}\right): 1698\right.$, 1610, 1207, 1081. Anal. Calcd. for $\mathrm{C}_{32} \mathrm{H}_{26} \mathrm{BrClN}_{2} \mathrm{O}_{4}$ : C, 62.20; H, 4.24; N, 4.53; Found: C, 62.49; H, 4.59; N, 4.70.

1-[2-(4-Chlorophenyl)-2-oxoethyl]-4-[[1-(ethoxycarbonyl)-3-(4-bromobenzoyl)]-indolizin-7yl]pyridinium bromide 5e. Orange crystals $(0.58 \mathrm{~g}, 86 \%$ yield $)$, m.p. $268-270{ }^{\circ} \mathrm{C}$. ${ }^{1} \mathrm{H}-\mathrm{NMR}$ : $9.97(1 \mathrm{H}, \mathrm{d}, \mathrm{H}-5$ ',$J=7.5 \mathrm{~Hz}), 9.41(2 \mathrm{H}, \mathrm{d}, \mathrm{H}-3, \mathrm{H}-5, J=6.6 \mathrm{~Hz}), 8.88\left(1 \mathrm{H}, \mathrm{d}, \mathrm{H}-8^{\prime}\right.$ ' $J=1,2$ Hz), 8.37 (2H, d, H-2, H-6, J=6.6 Hz), 8.18 (2H, d, H-10, H-14, J = 8.7 Hz), 7.80 (1H, s, H-2 ' ), $7.72\left(2 \mathrm{H}, \mathrm{d}, \mathrm{H}-12^{\prime}\right.$, H-16 ', J = $\left.7.5 \mathrm{~Hz}\right), 7.48$ (3H, ad, H-6', H-11, H-13), 7.30 (2H, d, H-13 ', $\left.\mathrm{H}-15^{\prime}, J=7.5 \mathrm{~Hz}\right), 7.29(2 \mathrm{H}, \mathrm{s}, 2 \mathrm{H}-7), 4.46\left(2 \mathrm{H}, \mathrm{q}, \mathrm{CH}_{2}, J=7.2 \mathrm{~Hz}\right), 1.44\left(3 \mathrm{H}, \mathrm{t}, \mathrm{CH}_{3}, J=7.2\right.$ 
Hz). IR (KBr, $v\left(\mathrm{~cm}^{-1}\right): 1707,1687,1639,1234,1203,1176$. Anal. Calcd. for $\mathrm{C}_{31} \mathrm{H}_{23} \mathrm{BrClN}_{2} \mathrm{O}_{4}$ : C, 54.53; H, 3.40; N, 4.10; Found: C, 54.49; H, 3.59; N, 4.40.

1-Ethyl 1'-methyl 3-benzoyl-3'-(4-methoxybenzoyl)-7,7'-biindolizine-1,1'-dicarboxylate 6 a. Yellow crystals $\left(0.30 \mathrm{~g}, 50 \%\right.$ yield), m.p. 293-294 ${ }^{\circ} \mathrm{C}$. $\mathrm{H}^{1}-\mathrm{RMN}: 10.04,9.97$ (2H, 2d, H-5 H-5 ', $J=7.2 \mathrm{~Hz}$ ), 8.79 (2H, as, H-8, H-8 ' ), 7.86 (6H, aq, H-2, H-2 ', H-12, H-16, H-12 ' , H-16 ' ), 7.487.63 (5H, m, H-13, H-15, H-14, H-6, H-6 ' ), 7.04 (2H, d, H-13 ', H-15 ' J = 8.8 Hz), 4.42 (2H, q, $\left.\mathrm{CH}_{2}, J=7.2 \mathrm{~Hz}\right), 3.95\left(3 \mathrm{H}, \mathrm{s}, \mathrm{OCH}_{3}\right), 3.91\left(3 \mathrm{H}, \mathrm{s}, \mathrm{OCH}_{3}\right), 1.44\left(3 \mathrm{H}, \mathrm{t}, \mathrm{CH}_{3}, J=7.2 \mathrm{~Hz}\right) . \mathrm{IR}$ $\left(\mathrm{KBr}, v\left(\mathrm{~cm}^{-1}\right): 1701,1685,1616,1263,1199,1174\right.$. Anal. Calcd. for $\mathrm{C}_{36} \mathrm{H}_{28} \mathrm{~N}_{2} \mathrm{O}_{7}$ : C, $71.99 ; \mathrm{H}$, 4.70; N, 4.66; Found: C, 72.15; H, 4.69; N, 4.95.

1-Ethyl 1'-methyl 3-(4-methoxybenzoyl)-3'-(4-bromobenzoyl)-7,7'-biindolizine-1,1'dicarboxylate 6 b. Yellow crystals $\left(0.34 \mathrm{~g}, 51 \%\right.$ yield), m.p. $350-352{ }^{\circ} \mathrm{C} .{ }^{1} \mathrm{H}-\mathrm{RMN}$ : 9.97 (2H, m, H-5, H-5'), 8.82 (2H, as, H-8, H-8 '), 7.82-7.88 (3H, m, H-2, H-12 ', H-16 ') 7.72-7.66 (3H, m, H-2, H-12, H-16) 7.52 (2H, m, H-6, H-6 '), 7.26 (2H, m, H-13 ', H-15 '), 7.05 (2H, d, H-13, H$15, J=8.2 \mathrm{~Hz}), 4.42\left(2 \mathrm{H}, \mathrm{q}, \mathrm{CH}_{2}, J=7.2 \mathrm{~Hz}\right), 3.95\left(3 \mathrm{H}, \mathrm{s}, \mathrm{OCH}_{3}\right), 3.92\left(3 \mathrm{H}, \mathrm{s}, \mathrm{OCH}_{3}\right), 1.43$ $\left(3 \mathrm{H}, \mathrm{t}, \mathrm{CH}_{3}, J=7.2 \mathrm{~Hz}\right)$. IR $\left(\mathrm{KBr}, \mathrm{v}\left(\mathrm{cm}^{-1}\right): 1734,1701,1643,1616,1232,1204,1147,1104\right.$. Anal. Calcd. for $\mathrm{C}_{36} \mathrm{H}_{27} \mathrm{BrN}_{2} \mathrm{O}_{7}$ : C, 63.63; H, 4.00; N, 4.12; Found: C, 63.85; H, 4.09; N, 4.35.

1-Ethyl 1'-methyl 3-(4-methylbenzoyl)-3'-(4-nitrobenzoyl)-7,7'-biindolizine-1,1'dicarboxylate 6c. Dark orange crystals $(0.31 \mathrm{~g}, 50 \%$ yield $)$, m.p. $319-321{ }^{\circ} \mathrm{C} .{ }^{1} \mathrm{H}-\mathrm{RMN}: 10.03$ (2H, at, H-5, H-5', J = 7.6 Hz), 8.83 (1H, s, H-8 ' ), 8.82 (1H, s, H-8), 8.40 (2H, d, H-13 ', H-15 ', $J=8.8 \mathrm{~Hz}), 7.99$ (2H, d, H-12 ', H-16 ', $J=8.8 \mathrm{~Hz}), 7.87(1 \mathrm{H}, \mathrm{s}, \mathrm{H}-2), 7.78$ (3H, ad, H-2, H-12, H-16), 7.59 (1H, dd, H-6 ', $J=7.2 \mathrm{~Hz}, J=1.6 \mathrm{~Hz}), 7.49(1 \mathrm{H}, \mathrm{dd}, \mathrm{H}-6, J=7.2 \mathrm{~Hz}, J=1.6 \mathrm{~Hz})$, $7.35\left(2 \mathrm{H}, \mathrm{d}, \mathrm{H}-13, \mathrm{H}-15, J=8 \mathrm{~Hz}, 4.43\left(2 \mathrm{H}, \mathrm{q}, \mathrm{CH}_{2}, J=7.2 \mathrm{~Hz}\right), 3.95\left(3 \mathrm{H}, \mathrm{s}, \mathrm{OCH}_{3}\right), 2.48,(3 \mathrm{H}\right.$, $\left.\mathrm{s}, \mathrm{CH}_{3}\right), 1.45\left(3 \mathrm{H}, \mathrm{t}, \mathrm{CH}_{3}, J=7.2 \mathrm{~Hz}\right)$. IR $\left(\mathrm{KBr}, v\left(\mathrm{~cm}^{-1}\right): 1718,1703,1643,1620,1525,1338\right.$, 1201, 1182, 1137, 1080. Anal. Calcd. for $\mathrm{C}_{36} \mathrm{H}_{27} \mathrm{~N}_{3} \mathrm{O}_{8}$ : C, 68.67; H, 4.32; N, 6.67; Found: C, $68.65 ; \mathrm{H}, 4.39 ; \mathrm{N}, 6.95$.

1-Ethyl 1'-methyl 3-(4-chlorobenzoyl)-3'-(4-methylbenzoyl)-7,7'-biindolizine-1,1'dicarboxylate $6 d$. Yellow crystals $(0.33 \mathrm{~g}, 54 \%$ yield $)$, m.p. $327-329{ }^{\circ} \mathrm{C} .{ }^{1} \mathrm{H}-\mathrm{RMN}: 10.02,10.01$ $\left(2 \mathrm{H}, 2 \mathrm{~d}, \mathrm{H}-5, \mathrm{H}-5^{\prime}\right.$ ' J = 5.2 Hz), 8.8 (2H, as, H-8, H-8 '), 7.87, 7.83 (2H, 2s, H-2, H-2 ' ), 7.80 (2H, d, H-12 ' , H-16 ' J = 8.4Hz), 7.77 (2H, d, H-12, H-16, J = 8 Hz), 7.52 (4H, aq, H-6 ', H-6, H-13, H-15), 7.35 (2H, d, H-13 ', H-15 ', J=8.4 Hz), $4.42\left(2 \mathrm{H}, \mathrm{q}, \mathrm{CH}_{2}, J=7.2 \mathrm{~Hz}\right), 3.95$ (3H, s, $\left.\mathrm{OCH}_{3}\right), 2.48\left(3 \mathrm{H}, \mathrm{s}, \mathrm{CH}_{3}\right), 1.44\left(3 \mathrm{H}, \mathrm{t}, \mathrm{CH}_{3}, J=7.2 \mathrm{~Hz}\right) . \mathrm{IR}\left(\mathrm{KBr}, \mathrm{v}\left(\mathrm{cm}^{-1}\right): 1705,1622,1217\right.$, 1083, 1147, 1045. Anal. Calcd. for $\mathrm{C}_{36} \mathrm{H}_{27} \mathrm{ClN}_{2} \mathrm{O}_{6}$ : C, 69.85; H, 4.40; N, 4.53; Found: C, 69.73; H, 4.39; N, 4.80.

1-Ethyl 1'-methyl 3-(4-bromobenzoyl)-3'-(4-chlorobenzoyl)-7,7'-biindolizine-1,1'dicarboxylate 6e. Yellow crystals $\left(0.33 \mathrm{~g}, 52 \%\right.$ yield), m.p. $334-335{ }^{\circ} \mathrm{C} .{ }^{1} \mathrm{H}-\mathrm{RMN}: 10.01(2 \mathrm{H}, \mathrm{d}$, H-5, H-5', J = 7.2 Hz), 8,83 (2H, ad, H-8, H-8 '), 7.82-7.70 (8H, m, H-2, H-2 ', H-12, H-16, H$12^{\prime}$, H-16 ', H-6, H-6' ), 7.53 (4H, m, H-13, H-15, H-13 ', H-15'), 4.42 (2H, q, CH,$J=7.2$ $\mathrm{Hz}), 3.95\left(3 \mathrm{H}, \mathrm{s}, \mathrm{OCH}_{3}\right), 1.43\left(3 \mathrm{H}, \mathrm{t}, \mathrm{CH}_{3}, J=7.2 \mathrm{~Hz}\right) . \mathrm{IR}\left(\mathrm{KBr}, \mathrm{v}\left(\mathrm{cm}^{-1}\right): 1705,1622,1217\right.$, 1083, 1147, 1045. Anal. Calcd. for $\mathrm{C}_{35} \mathrm{H}_{24} \mathrm{BrClN}_{2} \mathrm{O}_{6}$ : C, 61.46; H, 3.54; N, 4.10; Found: C, $61.63 ; \mathrm{H}, 3.40 ; \mathrm{N}, 4.28$. 


\section{Acknowledgements}

The authors gratefully thank to CNCSIS Bucharest (Grant IDEI_2023/2008) for financial support.

\section{References}

1. (a) Blewitt, H. L. Chem. Heterocycl. Compd. 1977, 30, 117. (b) Meiboroda, D. A.; Babaev, E. V.; Jug, K. J. Org. Chem. 1997, 62, 7100.

2. (a) Swinborne, J. H.; Klinkert, G. Advances in Heterocyclic Chemistry; Academic Press: London, 1979; Vol. 23, p 103. (b) Flitsch, W. Comprehensive Heterocyclic Chemistry; Pergamon: Oxford, 1984, Vol. 4, p 443.

3. (a) Malonne, H.; Hanuise, J.; Fontaine, J. Pharm. Pharmacol. Commun. 1998, 4, 241. (b) Gubin, J.; Luchetti, J.; Mahaux, J.; Nisato, D.; Rosseels, G.; Clinet, M.; Polster, P.; Chatlain, P. J. Med. Chem. 1992, 35, 981. (c) Kitadokoro, K.; Hagishita, S.; Sato, T.; Ohtani, M.; Miki, K. J. Biochem. 1998, 123, 619.

4. (a) Medda, S.; Jaisankar, P.; Manna, R. K.; Pal, B.; Giri, V. S.; Basu, M. K. J. Drug Target. 2003, 11, 123. (b) Bolle, L. D.; Andrei, G.; Snoeck, R.; Zhang, Y.; Lommel, A. V.; Otto, M.; Bousseau, A.; Roy, C.; Clercq, E. D.; Naesens, L. Biochem. Pharmacol. 2004, 67, 325. (c) Ebeid, M. Y.; El Moghazy Aly, S. M.; Hanna, M. M.; Romeih, F. A.; Barsoum, F. F. Bull. Fac. Pharm. 1997, 35, 171.

5. Campagna, F.; Carotti, A.; Casini, G.; Macripo, M. Heterocycles 1990, 31, 97.

6. (a) Olden, K.; Breton, P.; Grzegorzevski, K.; Yasuda, Y.; Gause, B. L.; Creaipe, O. A.; Newton, S. A.; White, S. L. Pharmacol. Ther. 1991, 50, 285. (b) Jaffrezou, J. P.; Levade, T.; Thurneyssen, O.; Chiron, M.; Bordier, C.; Attal, M.; Chatelain, P.; Laurent, G. Cancer Res. 1992, 52, 1352. (c) Artico, M.; Massa, S.; Stefancich, G.; Silvestri, R.; Di Santo, R.; Corelli, F. J. Heterocycl. Chem. 1989, 26, 503. (d) Bols, M.; Lillelund, V. H.; Jensen, H. H.; Liang, X. Chem. Rev. 2002, 102, 515. (e) Pearson, W. H.; Guo, L. Tetrahedron Lett. 2001, 42, 8267. (f) Asano, N.; Nash, R. J.; Molyneux, R. J.; Fleet, G. W. J. Tetrahedron: Asymmetry 2000, $11,1645$.

7. (a) Gubin, J.; Vogelaer, H.; Inion, H.; Houben, C.; Lucchetti, J.; Mahaux, J.; Rosseels, G.; Peiren, M.; Clinet, M.; Polster, P.; Chatelain, P. J. Med. Chem. 1993, 36, 1425. (b) Clive, D. L. J.; Coltart, D. M.; Zhou, Y. J. Org. Chem. 1999, 64, 1447. (c) Mynderse, J. S.; Fukuda, D. S. Eur. Pat. Appl. 133038 A2, 1985. (d) Chatelain, P.; Laruel, A.; Beaufort, P.; Meysmans, L.; Clinet, M. Cardioscience 1992, 3, 117.

8. (a) Ruprecht, R. M.; Mullaney, S.; Andersen, J.; Bronson, R. J. Acquired Immune Defic. Syndr. 1989, 2, 149. (b) Gruters, R. A.; NeefJes, J. J.; Tersmette, M.; De Goede, R. E. Y.; Tulp, A.; Huisman, H. G.; Miedema, F.; Ploegh, H. L. Nature 1987, 330, 74. (c) Kaspas, A.; 
Fleet, G. W. J.; Dwek, R. A.; Petursson, S.; Namgoong, S. K.; Ramsden, N. G.; Jacob, G. S.; Radamacher, T. W. Proc. Natl. Acad. Sci.U.S.A. 1989, 86.

9. (a) Saeva, F. D.; Luss, H. R. J. Org. Chem. 1988, 53, 1804. (b) Fletetcher, G. L.; Bender, S. L.; Wadsworth, D. H. U.S. Patent 4577024 1986. (c) Sonnenschein, H.; Henrich, G.; ReschGenger, V.; Schulz, B. Dyes Pigments 2000, 46, 23. (d) Vlahovici, A.; Andrei, M.; Druta, I. J. Lumin. 2002, 96, 279. (e) Vlahovici, A.; Druta, I.; Andrei, M.; Cotlet, M. J. Lumin. 1999, 82, 155. (f) Rotaru, A. V.; Druta, L. D.; Oeser, T.; Mueller, T. J. J. Helv. Chim. Acta 2005, 88, 1798. (g) Delattre, F.; Woisel, P.; Surpateanu, G.; Cazier, F.; Blach, P. Tetrahedron 2005, $61,3939$.

10. Padwa, A. 1,3-Dipolar Cycloaddition Chemistry, John Wiley \& Sons: New York, 1984.

11. (a) Broggini, G.; Zecchi, G. Synthesis 1999, 905. (b) NáJera, C.; Sansano, J. M. Curr. Org. Chem. 2003, 7, 1105. (c) Padwa, A.; Austin, D. J.; Precedo, L.; Zhi, L. J. Org. Chem. 1993, $58,1144$.

12. (a) Druta, I.; Dinica, R.; Bacu, E.; Humelnicu, I. Tetrahedron 1998, 54, 10811. (b) Druta, I.; Cuciac, C.; Blanaru, C.; Avram, E. Ann. St. Univ. "Al. I. Cuza" Iasi 2001, 9, 109. (c) Rotaru, A. V.; Danac, R. P.; Druta, I. D. J. Heterocycl. Chem. 2004, 41, 893.

13. Angulo, G.; Grampp, G.; Rosspeintner, A. Spectrochimica Acta, Part A 2006, 65, 727. 